\title{
BMJ Open Protocol for a prospective, longitudinal, cohort study of recovery pathways, acute biomarkers and cost for children with persistent postconcussion symptoms: the Take CARe Biomarkers study
}

Michael Takagi, ${ }^{1,2}$ Franz E Babl, ${ }^{1,3,4}$ Nicholas Anderson, ${ }^{1}$ Silvia Bressan, ${ }^{1,5}$ Cathriona J Clarke, ${ }^{1}$ Ali Crichton, ${ }^{1}$ Kim Dalziel, ${ }^{6}$ Gavin A Davis, ${ }^{1,7}$ Melissa Doyle, ${ }^{1,2}$ Kevin Dunne, ${ }^{1,3,8}$ Celia Godfrey, ${ }^{1,3}$ Stephen J C Hearps, ${ }^{1}$ Vera Ignjatovic, ${ }^{1,3}$ Georgia Parkin, ${ }^{1}$ Vanessa Rausa, ${ }^{1}$ Marc Seal, ${ }^{1}$ Emma Jane Thompson, ${ }^{1}$ Katie Truss, ${ }^{1,2}$ Vicki Anderson ${ }^{1,2,3,9}$

To cite: Takagi M, Babl FE, Anderson N, et al. Protocol for a prospective, longitudinal, cohort study of recovery pathways, acute biomarkers and cost for children with persistent postconcussion symptoms: the Take CARe Biomarkers study. BMJ Open 2019;9:e022098. doi:10.1136/ bmjopen-2018-022098

- Prepublication history for this paper is available online. To view these files, please visit the journal online (http://dx.doi org/10.1136/bmjopen-2018022098).

Received 22 February 2018 Revised 20 September 2018 Accepted 20 November 2018

Check for updates

(C) Author(s) (or their employer(s)) 2019. Re-use permitted under CC BY-NC. No commercial re-use. See rights and permissions. Published by BMJ.

For numbered affiliations see end of article.

Correspondence to

Dr Franz E Babl;

franz.babl@rch.org.au

\section{ABSTRACT}

Introduction The majority of children who sustain a concussion will recover quickly, but a significant minority will experience ongoing postconcussive symptoms, known as postconcussion syndrome (PCS). These symptoms include emotional, behavioural, cognitive and physical symptoms and can lead to considerable disability. The neurobiological underpinnings of PCS are poorly understood, limiting potential clinical interventions. As such, patients and families frequently re-present to clinical services, who are often ill equipped to address the multifactorial nature of PCS. This contributes to the high cost of concussion management and the disability of children experiencing PCS. The aims of the present study are: (1) to plot and contrast recovery pathways for children with concussion from time of injury to 3 months postinjury, (ii) evaluate the contribution of acute biomarkers (ie, blood, MRI) to delayed recovery postconcussion and (3) estimate financial costs of child concussion to patients attending the emergency department (ED) of a tertiary children's hospital and factors predicting high cost.

Methods and analysis Take C.A.Re is a prospective, longitudinal study at a tertiary children's hospital, recruiting and assessing 525 patients aged $5-<18$ years (400 concussion, 125 orthopaedic injury) who present to the ED with a concussion and following them at 1-4 days, 2 weeks, 1 month and 3 months postinjury. Multiple domains are assessed: preinjury and postinjury, clinical, MRI, blood samples, neuropsychological, psychological and economic. PCS is defined as the presence of $\geq 2$ symptoms on the Post Concussive Symptoms Inventory rated as worse compared with baseline 1 month postinjury. Main analyses comprise longitudinal Generalised Estimating Equation models and regression analyses of predictors of recovery and factors predicting high economic costs.

Ethics and dissemination Ethical approval has been obtained through the Royal Children's Hospital Melbourne Human Research Ethics Committee (33122). We aim to disseminate the findings through international

\section{Strengths and limitations of this study}

This study will recruit and follow-up children with concussion from acute emergency department presentation to 3 months, reducing potential bias related to later recruitment.

- The breadth and depth of the domains assessed throughout the study are extensive, particularly the biomarkers (ie, brain MRI and blood).

- The present study is based in an emergency department, which may limit the generalisability of the findings to other clinical settings.

conferences, international peer-reviewed journals and social media.

Trial registration number ACTRN12615000316505; Results.

\section{INTRODUCTION}

Concussion can be defined as a traumatic brain injury (TBI) induced by biomechanical forces, resulting in the rapid onset of shortlived impairment of neurological function that resolves spontaneously. ${ }^{1}$ During childhood and adolescence, concussions (often used synonymously with mild TBI) are a common occurrence and constitute a significant public health concern. Indeed, approximately $90 \%$ of head injury presentations at emergency departments (EDs) are classified as mild, ${ }^{2}{ }^{3}$ and Meehan and Mannix ${ }^{4}$ found that approximately 144000 paediatric patients present to EDs each year with a concussion in the USA.

In addition to the postinjury sequelae, concussions represent a substantial cost to the 
medical system. Recent epidemiological data highlight that while moderate and severe TBI are more expensive at the individual level, at the population level, healthcare costs following concussion far exceed those of moderate and severe TBI. ${ }^{5}$ For example, in the USA, Graves $e t a l^{5}$ examined the overall cost of paediatric mild TBI (mTBI) in a nationwide cohort of 20 million people (308174 with $\mathrm{mTBI}$ ). The authors estimated that the total population-level costs for paediatric mTBI in the first 3 months postinjury was US $\$ 695$ million.

Fortunately, the majority of children return to preinjury levels of functioning relatively quickly after sustaining a concussion. ${ }^{16}$ However, approximately one-third of children continue to experience symptoms for an extended period postinjury ${ }^{7}$ (eg, beyond 1 month), which is frequently referred to as postconcussion syndrome (PCS). Despite an increased research focus on paediatric concussion in recent years, the mechanisms underpinning PCS remain poorly understood.

PCS refers to a constellation of somatic, cognitive, psychological and behavioural symptoms, ${ }^{7}$ and a range of child/family and clinical factors have been found to be associated with prolonged recovery. For example, female sex, age, migraine history, previous concussion and the experience of specific symptoms (headache, sensitivity to noise, fatigue and answering questions more slowly) is associated with worse outcome. ${ }^{7}$ Psychosocial factors (eg, depression, anxiety) have also been associated with delayed recovery postconcussion. ${ }^{8}$ However, the bulk of paediatric concussion studies have investigated older adolescents, with very few studies considering children younger than 12 years, so it is unclear if these findings generalise to the full clinical population.

In a recent large multicentre study, Zemek et al derived and validated a risk score to predict PCS in children assessed in the ED following acute concussion ${ }^{7}$; however, its correlation with recovery trajectories or its value for clinical management or tailored treatment recommendations is yet to be determined. Further, the score was derived primarily from demographic and acute medical data, which may provide too narrow a scope to understand the factors underpinning recovery. Recent evidence indicates that the biomechanical force transmitted to the brain during a concussion results in a 'metabolic cascade' of ionic shifts, altered metabolism, impaired connectivity and neurotransmission changes, ${ }^{9}$ as well as microscopic axonal injury. ${ }^{10}$ Research to improve long-term care of paediatric concussion depends on early identification of those most in need of intervention. There is growing optimism that delayed metabolic recovery and subtle brain pathology are key to understanding prolonged postconcussive problems and for early detection of children at risk who require early intervention. Indeed, a recent systematic review published by our group ${ }^{11}$ highlights the potential utility of several blood biomarkers (eg, glial fibrillary acidic protein (GFAP), ubiquitin C-terminal hydrolase-L1 (UCH-L1)). Measurement of these mechanisms and their interplay, via blood biomarkers and sophisticated neuroimaging, may provide objective indices of injury severity, assist in identifying children at risk for delayed recovery, help define injury clinical phenotypes and inform decisions regarding safe return to normal activities.

\section{Aims}

The study aims are as follows.

1. To evaluate the contribution of acute biomarkers to delayed recovery postconcussion, tapping the key aspects of the postinjury metabolic cascade and associated brain pathology to better understand the mechanisms underlying delayed symptom recovery.

2. To plot and contrast recovery pathways for children with concussion compared with orthopaedic injury (OI) from time of injury to 3 months postinjury in four domains: postconcussive symptoms, cognition, physical function and mental health and quality of life.

3. To estimate financial costs of child concussion to patients attending the ED of a tertiary children's hospital and factors predicting high cost.

\section{METHODS AND ANALYSIS}

\section{Patient and public involvement}

For the initial pilot phase of Take Concussion Assessment and Recovery Research (CARe) study, anonymous participant feedback was sought at each stage to identify participant concerns and priorities and to gain a better understanding of their experience. For example, identifying what specific information the partipants wanted during clinical feedback (eg, should my child wear a helmet to play football?). The feedback was incorporated into the design of Take CARe Biomarkers. Participants were not involved in the recruitment and conduct of the study. The results of the study will be disseminated to study participants via individual letters.

\section{Study design}

The present study extends on The Take CARe study (see Bressan $e a^{12}$ ) protocol and is henceforth referred to as Take CARe Biomarkers. Take CARe Biomarkers incorporates the elements from the original study and substantially expands and adds novel aspects (eg, MRI, blood biomarkers and extensively expanded clinical measures). As such, it can be considered a separate study as opposed to an addendum to Take CARe. It is a single-centre, prospective, longitudinal cohort study of children with concussion and orthopaedic injuries and their parents. Children are followed for 3 months postinjury and assessed within 48 hours (T0), 1-4 days (T1), 2 weeks (T2), 1 month (T3) and 3 months (T4) postinjury. The study has three methods of data collection: in-person, online and by telephone based on patient and family preference and with the purpose of optimising compliance to completion of relevant study procedures. The in-person method will be detailed in this paper. Participants enrolled in the online and telephone paths complete only the questionnaire portion of the study at each time point, providing informative data for aims (1), 
(2) and (3). These participants are closely monitored and are invited to attend the concussion clinic if their symptoms persist or worsen. In-person participants also have the option of completing the full study protocol or a reduced protocol omitting MRI and/or blood collection. Because of the additional measures, the participants recruited for Take CARe Biomarkers are a separate cohort relative to the original Take CARe study. On establishing the Take CARe Biomarkers protocol, recruitment for Take CARe has ceased.

\section{Study setting}

Take CARe Biomarkers is conducted at the Murdoch Children's Research Institute (MCRI) and Royal Children's Hospital (RCH), in Melbourne, Australia. Patient recruitment and initial assessments take place in the RCH ED. Follow-up appointments occur in an outpatient concussion clinic at MCRI, as well as the Medical Imaging and Pathology departments of the RCH. The concussion clinic is a collaboration between RCH clinicians and MCRI clinical researchers.

\section{Definitions}

Concussion is defined according to the Consensus Statement on Concussion in Sport put forth by the Concussion in Sport Group ${ }^{13}$ as a blunt head injury caused by biomechanical forces including one or more symptoms in one or more of the following categories: somatic (eg, headache, vomiting), cognitive (eg, feeling like in a fog), emotional (eg, lability), physical signs (eg, loss of consciousness, amnesia), behavioural changes (eg, irritability); cognitive impairment (eg, slowed reaction times) and/or sleep disturbance (eg, insomnia).

PCS is defined as clinically significant symptoms persisting beyond 1 month postinjury (see the Methodological considerations section for further explanation of PCS definition).

\section{Study population \\ Concussion \\ Inclusion criteria}

Patients aged 5-18 years presenting to the ED with a concussion sustained within the previous 48 hours are screened for eligibility.

\section{Exclusion criteria}

Patients with any of the following are excluded from participation: Glasgow Coma Scale (GCS) <13, structural/haemorrhagic intracranial injury, clinical evidence of a cerebrospinal fluid leak.

\section{Orthopaedic injury}

\section{Inclusion criteria}

Patients aged 5-18 years presenting to the ED with an isolated, upper extremity fracture are screened for eligibility.

\section{Exclusion criteria}

Patients with any of the following are excluded: evidence of head injury or concussion; hypoxia, hypotension or shock and needing sedation (eg, sedative medication for fracture manipulation; fentanyl alone is an exception if the assessment occurred $60 \mathrm{~min}$ post-fentanyl administration)

\section{General exclusion criteria}

Patients with the following are excluded regardless of group (concussion/OI): developmental or intellectual disability; history of moderate or severe traumatic brain injury (TBI) or neurological conditions; needing intubation, general anaesthesia or neurosurgical intervention; abdominal, chest, back or neck injury; history of psychosis or bipolar disorder; medication use (antidepressants, stimulants); intoxication on presentation to the ED; fever; injury resulting from abuse or assault; multiple injuries; no clear history of trauma as the primary event; insufficient understanding of English and currently enrolled in the study.

\section{Patient selection and recruitment}

Patients who present to the RCH ED, are screened by trained research assistants (RA) daily (weekdays: 09:00 to 22:00, weekend: 12:00 to 22:00) by reviewing the $\mathrm{RCH}$ ED visits software. The treating physician is contacted to confirm the patient is eligible and meets the study criteria. If eligibility is confirmed, families are approached to participate in the in-person programme (concussion and orthopaedic group) or the online or telephone completion method (concussion group only). As part of the in-person programme, there is the option to complete an MRI (one time point, 2 weeks) and collection of venous blood samples (all time points). If the in-person programme is refused, the online or phone option is offered to the concussion group only. Informed consent to participate is obtained from parents and children for all study completion methods. A record of all patients screened, exclusion criteria and reason for refusal is recorded by the RA.

\section{Initial assessment in the ED}

Once consent is obtained, all participants (concussion and orthopaedic controls) are assessed in the ED. Injury-related information is provided by treating doctors using a dedicated clinical report form detailing injury mechanism, clinical findings and ED management. Preinjury and demographic information is provided by parents in the ED.

Parents and children complete the Post-Concussion Symptom Inventory (PCSI), a developmentally appropriate scale that measures both preinjury and current physical, cognitive, emotional and sleep-related symptoms. Parents complete the parent-report form. The self-report form is completed by children older than 13 years, and children aged 5-12 years complete the PCSI for Children (PCSI-C) by interview or self-report. The PCSI has shown good reliability and validity. ${ }^{14}$ In the ED, patients also complete the Sports Concussion Assessment Tool third Edition (SCAT3), ${ }^{15}$ under 
supervision of an RA. See Bressan et $a l^{12}$ for a more detailed description of the SCAT3. In brief, the SCAT3 is a multimodal test battery for the assessment of acute concussion symptoms. It includes a version for children aged 13 years and over and a separate version for children aged 5-12 years (Child-SCAT3). The SCAT3 comprises a symptom checklist, a brief cognitive assessment of orientation, immediate and delayed recall and concentration and a physical evaluation of balance and coordination. Recruitment for the present study initiated prior to the publication of the SCAT5 $5^{16}$ and Child SCAT $5,{ }^{17}$ and therefore we continued to use SCAT3 and Child SCAT3 for the study.

Acute pain is reported in the ED through completion of the Faces Pain Scale-Revised ${ }^{18}$, which is a validated self-report measure of pain in children aged five years or older. If participants have consented to provide a venous blood sample, the treating doctor or nurse collects $1.4 \mathrm{~mL}$ of blood to be analysed by Haematology Research laboratory staff.

\section{Follow-up assessments}

Follow-up assessments take place in the concussion clinic 1-4 days, 2 weeks, 1 month and 3 months postinjury. Participants were sent an email with a link to an online database where they complete the PCSI daily until their follow-up appointment. They are also sent a link once between the 1-4 days and 2-week appointments (ie, 1 week postinjury). Clinic consultations are conducted by trained RAs and participants are assessed by a clinician (eg, neuropsychologist, rehabilitation physician) with expertise in concussion management. All patients attend 1-4 day and 2-week appointments in-person and symptom severity/burden is assessed by the PCSI. Patients determined by a clinician to have returned to preinjury levels of functioning at 2 weeks postinjury complete the 1 month assessment measures online, while those who remain symptomatic (as measured by the PCSI) attend the concussion clinic at 1 month. All patients return to the clinic for follow-up assessments at 3 months (see figure 1). In addition, orthopaedic patients attend all appointments T1-T4 onsite.

\section{Study outcomes}

1. The primary outcome for aim 1 is the proportion of children aged 5-18 years who continue to experience ongoing concussion symptoms at 2 weeks and 1 month postinjury. This time point was chosen for clinical reasons as a substantial proportion of children recover or largely recover within 2 weeks and for those that do not, many seek intervention at this time. Ongoing symptoms are defined as the presence of two or more symptoms on the PCSI rated as worse compared with baseline. $^{19}$

2. The biomarkers component of the study is exploratory. With that in mind, the primary outcome for aim 2 is ongoing postconcussion symptoms at 2 weeks postin- jury as predicted by biomarkers (ie, blood and MRI). The specific biomarkers that best predict ongoing concussion symptoms will be derived using statistical modelling.

3. The primary outcome for aim 3 is the overall cost of paediatric concussion, which includes costs associated with medical treatment, hospital stays and costs to the patient and family (eg, lost days of work).

\section{Secondary outcomes}

Secondary outcomes include (1) the 1-4 days, 1 month and 3 month performance in the following domains: neurocognition, physical recovery, psychosocial/ mental health status, quality of life, post-traumatic stress symptoms, family burden; (2) preinjury/injury-related (including blood biomarkers, MRI) and early postinjury factors associated with rapid or prolonged recovery and (iii) timing of return to school/return to sport.

\section{Data collection and research measures}

A summary of data collection at each time point is provided in table 1 .

\section{Clinical information and demographics}

Child and parent demographic information including age, sex, level of education, marital status, work status and ethnic background are reported by parents in the ED. Clinically relevant preinjury information including medical and psychiatric history, prior concussive injuries, hand dominance, engagement in sport, sleep disturbance and history of frequent vomiting are also provided by parents. Retrospective rating of PCS to determine baseline symptomology is also collected using the PCSI in the ED.

Injury-related information is obtained using a dedicated clinical record form completed by treating doctors. For children with concussion, injury-related information collected includes: the date and time of the injury, date and time of triage assessment, time seen by clinician, description of the presenting injury, diagnosis, if the patient was placed in a neck collar, presence/absence of clinical CT scan, symptoms, mechanism of injury, general examination (ie, GCS, headache, location of head strike), activities participated in since the injury (eg, school or sport), admission and discharge status (to the ED Short Stay Unit, or home). For children with orthopaedic injuries, injury-related information collected includes: the date and time of the injury, date and time of triage assessment, time seen by clinician, description of the presenting injury, presence/absence of sedative medication, diagnosis, if there was any evidence of a concussion, hypoxia, hypotension or shock during or following the injury, the mechanism of injury, type of injury, location of injury and the treatment given.

Post-concussive symptom evaluation

Post-concussive symptoms are assessed using the PCSI and SCAT3/Child-SCAT3. 


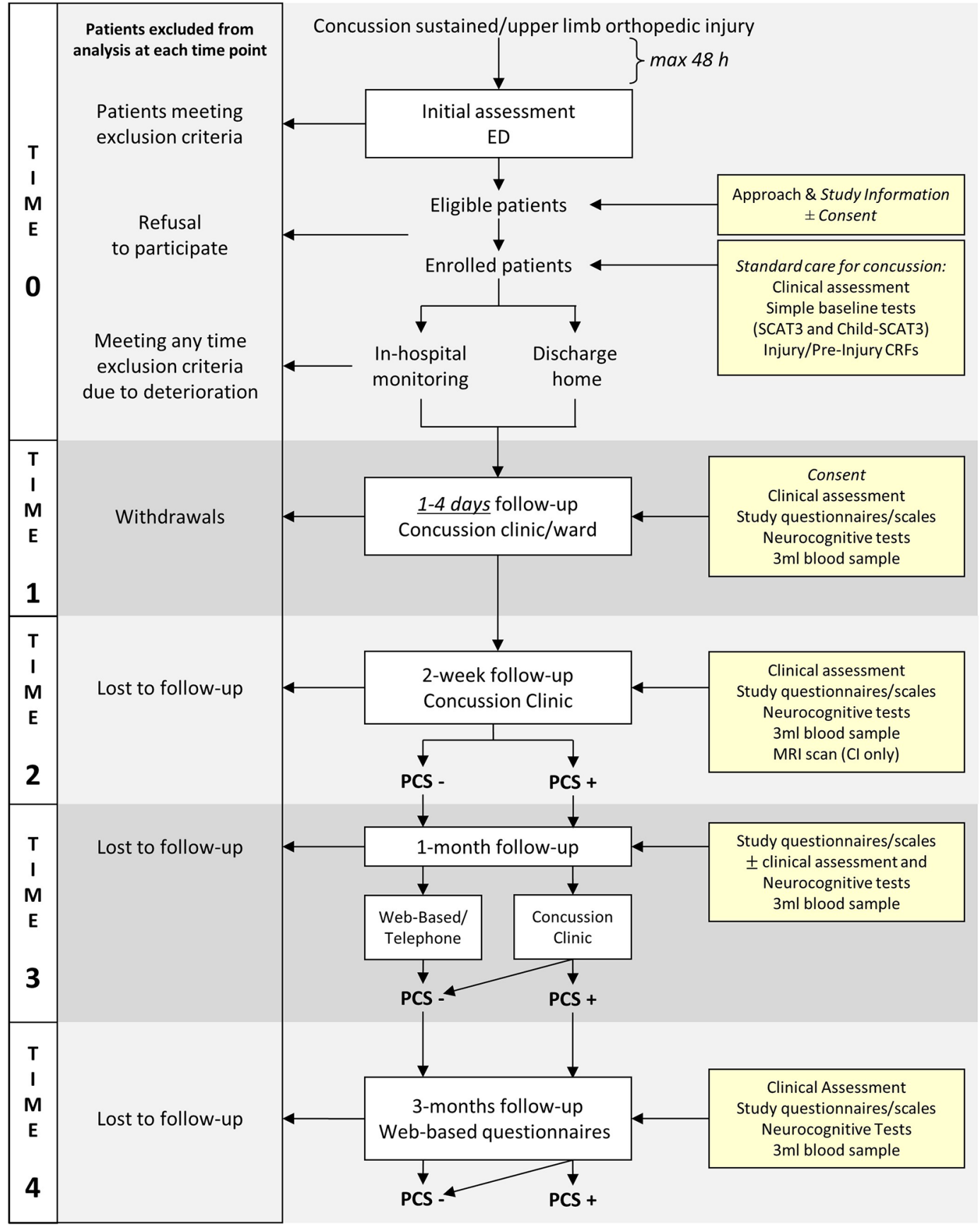

Figure 1 A flow chart of study procedures.

\section{Physical domain evaluation}

Balance and coordination are assessed at each time point using the SCAT3.

\section{Blood samples}

With participant consent, $1.4 \mathrm{~mL}$ of venous blood is collected from children in the ED and at each follow-up appointment. Once all data is collected, the proteomic based blood biomarker study will occur in two phases, discovery and validation, with comparison between concussion and orthopaedic injured samples in each phase. The discovery phase (30 concussion +30 orthopaedic samples), will involve: i. Targeted testing of biomarkers previously shown to be important in concussion and additional general markers of inflammation. This component will use commercially available Millipore multiplex panels, cytokine (interleukin (IL)-1b, IL-6, IL-8, IL-10, tumour necrosis factor alpha); soluble intercellular adhesion molecule-1 (sICAM-1), soluble vascular cell adhesion molecule-1 (sVCAM-1)); sE-selectin; neurogenerative (S100B, amyloid beta 1-40, amyloid beta 1-42) and neurological (neuron-specific enolase (NSE), Tau (Thr231), Tau (total)), GFAP.

ii. Untargeted testing of all proteins detectable in plasma. This component will use a recently established 


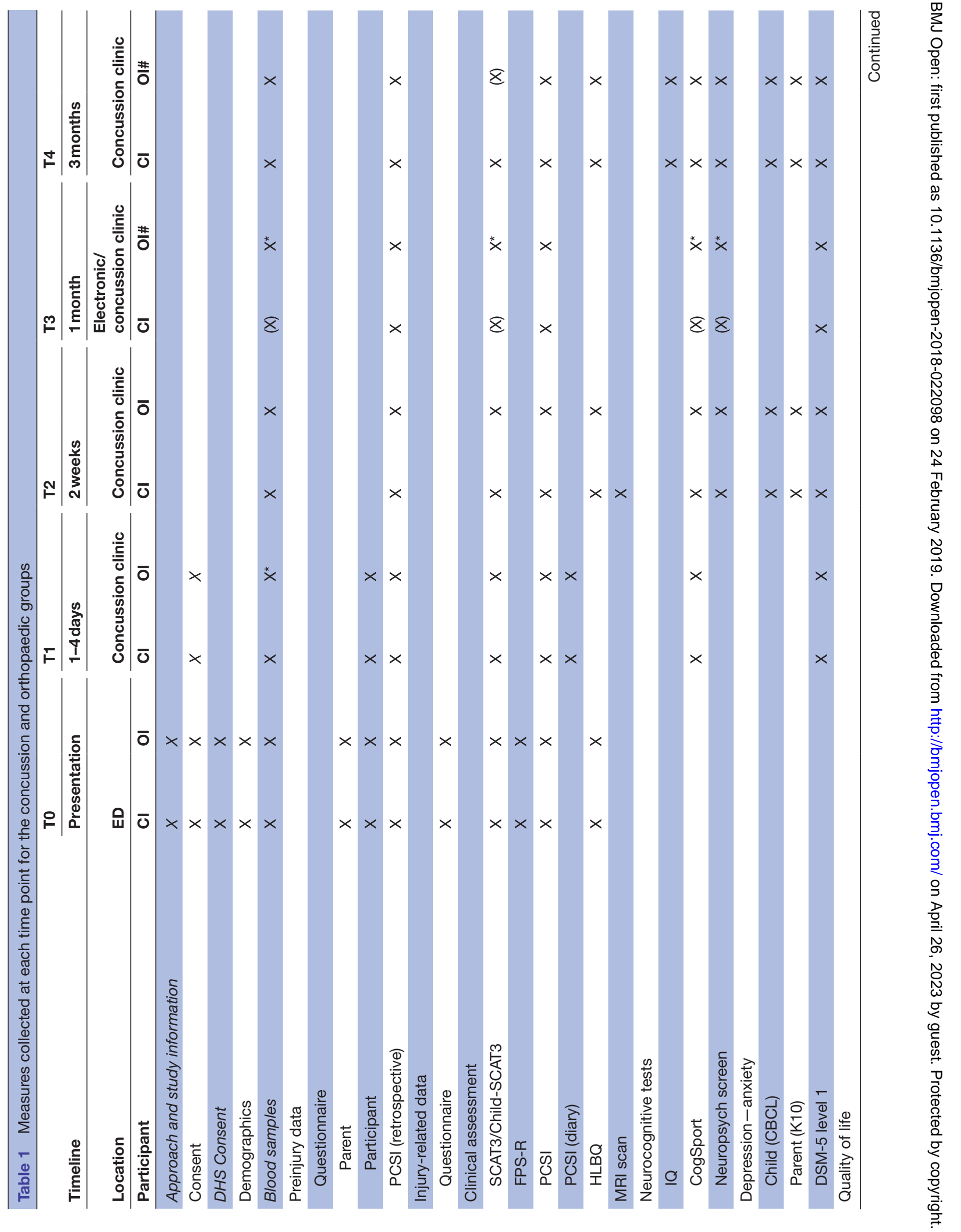




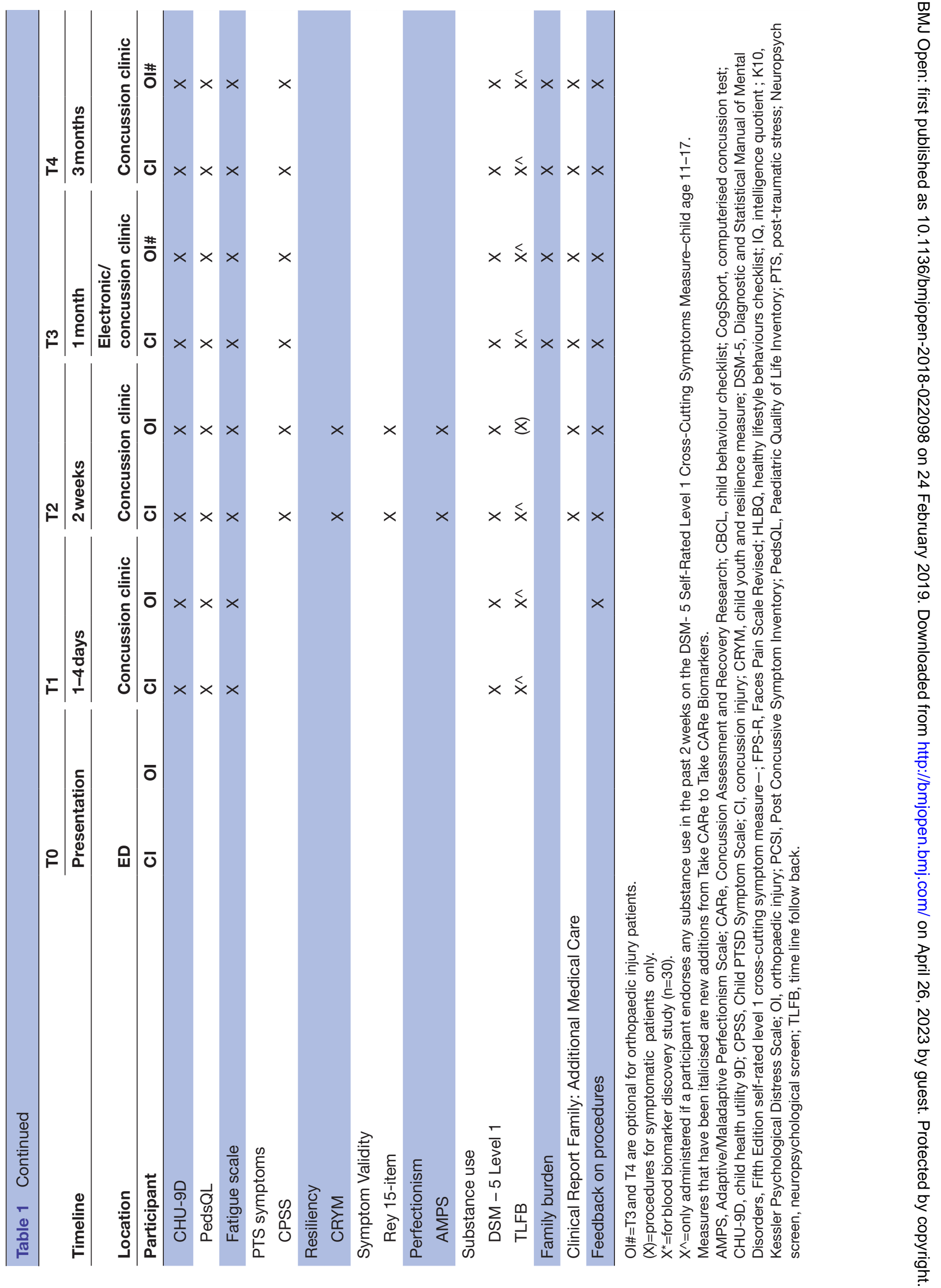


mass spectrometry approach, sequential window acquisition of all theoretical mass spectra which assesses changes in relative expression of up to 400 proteins per sample. This will allow for exploration of potentially clinically relevant proteins not yet examined in concussion research.

The validation phase will involve all samples in the cohort. Specifically, differences in concentration of proteins of interest identified as potential biomarkers in the discovery phase will be validated using ELISA test kits. This is a standard approach used for biomarker validation.

Our multiple sampling will provide novel data documenting changes over time in blood biomarkers and reflecting recovery of metabolic status which will be used to contribute to decision making on safe return to sports.

\section{MRI}

Structural and functional MRI are conducted on concussion sample of 50 participants at the 2-week concussion clinic follow-up appointment. Scans are conducted using a 3-Tesla Siemens Trio MRI scanner (Siemens Medical Solutions, Erlangen, Germany). The MRI protocol are optimised for the assessment of the paediatric brain and will comprise T1 anatomical (MPRAGE MoCo; $\mathrm{TR}=2530 \mathrm{~ms}$, TE $1=1.77 \mathrm{~ms}$, TE2 $=3.51 \mathrm{~ms}, \mathrm{TE} 3=5.32 \mathrm{~ms}$, TE4 $=7.2 \mathrm{~ms}$, voxel $\left.=0.9 \mathrm{~mm}^{3}\right)$, susceptibility weighted imaging (SWI; TR=48 ms, TE1=12.30 ms, TE2=39.36 $\mathrm{ms}$, voxel $=0.9 \times 0.7 \times 1.0 \mathrm{~mm})$, diffusion weighted imaging (60 diffusion gradient directions, b-value $=2800 \mathrm{~s}$ / $\mathrm{mm}^{2}, 2.4 \times 2.4 \times 2.4 \mathrm{~mm}^{3}$ isotropic voxel size, $\mathrm{TE}=110 \mathrm{~ms}$, $\mathrm{TR}=3200 \mathrm{~ms}$, four volumes without diffusion weighting $\mathrm{b}=0 ; 25$ diffusion gradient directions, $\mathrm{b}-\mathrm{value}=1100 \mathrm{~s}$ / $\mathrm{mm}^{2}, 2.4 \times 2.4 \times 2.4 \mathrm{~mm}^{3}$ isotropic voxel size, TE= $110 \mathrm{~ms}$, $\mathrm{TR}=3200 \mathrm{~ms}$, four volumes without diffusion weighting $\mathrm{b}=0$ ), dual inversion recovery (DIR; TR $=7400 \mathrm{~ms}, \mathrm{TE}=324$ $\mathrm{ms}$, voxel $=1.1 \mathrm{~mm}^{3}$ ), arterial spin labelling (pcASL; $\mathrm{TR}=3370 \mathrm{~ms}, \mathrm{TE}=18 \mathrm{~ms}$, voxel $=2.5 \times 2.5 \times 3.0 \mathrm{~mm}$, post-labelling delay $=1600 \mathrm{~ms}$ ) and resting state data EPI BOLD (ic-fMRI; multiband factor $=3, \mathrm{TR}=39800 \mathrm{~ms}, \mathrm{TE}=33 \mathrm{~ms}$, voxel $=2.5 \mathrm{~mm}^{3}$ ). The protocol contains the gold standard sequences validated by the Human Connectome Project, ${ }^{16}$ which include multiband accelerated echoplanar imaging sequences designed to maximise the spatial and temporal resolution of MR data. MRI data will contribute information that will be diagnostic postinjury (SWI and DIR), allow measurement of changes in cerebral blood perfusion (ASL) and brain function (ic-fMRI) at 2 weeks postinjury, and detailed analysis of any disruption to neural circuitry (DWI) that may underpin PCS.

\section{Neuropsychological assessment}

The neuropsychological measures described in the Take CARe protocol have been included in Take CARe Biomarkers (see Bressan et $a l^{12}$ for more details). This includes CogSport, ${ }^{20}$ a widely used computerised neuropsychological test that assesses processing speed, attention, learning and working memory. Additional neuropsychological tests included in this study include the following.

\section{Estimate of general intellectual ability}

Wechsler scales are used assess intellectual ability of participants, using the full scale intellectual quotient (IQ). The age-appropriate IQ is important to adequately characterise the sample, and an estimation of IQ provides the context to interpret all measures. Wechsler scales are administered at 3 months postinjury: the Wechsler Abbreviated Intelligence Scale-II (WASI-II) ${ }^{21}$ for children aged 7-18 years and the Wechsler Preschool and Primary Scale of Intelligence-IV (WPPSI-IV) ${ }^{22}$ for children aged 5-7 years. The two subtest form of the WASI and WPPSI is administered (ie, matrix reasoning and vocabulary).

\section{Neuropsychological screen}

A neuropsychological screen is administered at 2 weeks, 1 month (if symptomatic) and at 3 months. Measures are selected which (1) covered the age range of the study; (2) assessed cognitive domains previously identified as vulnerable in concussion and (3) have minimal practice effects. Working memory, attention and processing speed is assessed at 2 weeks, 1 month (if symptomatic), and 3 months, executive function will be assessed at $\mathrm{T} 4$ only.

\section{Information processing}

The working memory and processing speed indices from the Wechsler scales are used to assess information processing. For participants aged 7-18 years, the coding and digit span subtests from the Wechsler Intelligence Scale for Children-Fourth Edition (WISC-IV) ${ }^{23}$ are used as measures of processing speed and working memory, respectively. For participants aged 5-7 years, the working memory subtests (eg, picture memory) and processing speed subtests (eg, bug search) from the WPPSI-IV ${ }^{22}$ are used to assess working memory and processing speed.

\section{Attention}

The Contingency Naming Test ${ }^{24}$ assesses aspects of executive function, such as processing speed and inhibitory attention. It is suitable for use in children aged 7 years and older. ${ }^{25}$ There are four tasks based on a series of coloured shapes within a shape: (1) name the colour of the shape, (2) name the outside shape, (3) if the inside and outside shape match name the colour, if the inside and outside shape are different name the outside shape (4) same as rule 3 , however, the rule is reversed if an arrow appears above the coloured shape. Time to completion, self-corrections and errors are recorded by the examiner.

\section{Memory and learning}

The Rey Auditory Verbal Learning is a list learning task that is a measure of verbal memory and learning ability. ${ }^{26}$ The list has 15 items that are read out to the participant. Recall is tested over five consecutive learning trials. Administration time is $5 \mathrm{~min}$ with a delayed recall component of $20 \mathrm{~min}$, and is standardised for use in children 7 years and older. 


\section{Executive function}

The trail-making test is a measure of attention, visual search ability, motor function and mental flexibility. ${ }^{27}$ This test involves connecting circles with numbers or letters in sequential order by drawing lines with a pencil. There are two tests, one with only numbers in order and the second to alternate between numbers and letters in alphabetical order. Scores are obtained from the time taken to complete the task and the number of errors made. This test has been standardised for children 7 years and older and an abbreviated version was given to children aged 5 and 6 years. ${ }^{27}$

The Controlled Oral Word Association Test ${ }^{28}$ examines verbal fluency and the purpose of the test is to evaluate the spontaneous production of words within a limited amount of time. Participants are asked to produce orally as many words as possible, beginning with a given letter of the alphabet. For the present study, the letters are ' $\mathrm{F}$ ', ' $\mathrm{A}$ ' and ' $\mathrm{S}$ '. Three trials are administered (ie, one for each letter) and participants have $60 \mathrm{~s}$ to generate words. Participants are instructed to avoid saying proper nouns (eg, Bob, Brylcreem) or saying the same word using a different ending (eg, run, running). Scoring for this task involves the number of words for each letter, the total number of words and the number of rule breaks. ${ }^{27}$ This task has been standardised for use in children 7 years and older.

\section{Psychosocial evaluation}

The psychosocial measures described in the Take CARe protocol have been included this study (see Bressan $e t l^{12}$ for more details). This includes the child behaviour checklist $^{29}$, the Child Post-Traumatic Stress Symptom Scale ${ }^{30}$, the Paediatric Quality of Life Inventory ${ }^{31}$, the Kessler Psychological Distress Scale ${ }^{32}$ and a measure of family burden. Additional measures include the following.

\section{Mental health: Diagnostic and Statistical Manual of Mental Disorders, Fifth Edition (DSM-5) Self-Rated Level 1 Cross-Cutting Symptoms Measure}

The DSM-5 Self-Rated Level 1 Cross-Cutting Symptom Measure-child age $11-17$ years ${ }^{33}$ is a self-rated measure that assesses mental health domains that are important across psychiatric diagnosis. The measure consists of 25 questions that assess 12 psychiatric domains, including depression, anger, irritability, mania, anxiety, somatic symptoms, inattention, suicidal ideation/attempt, psychosis, sleep disturbance, repetitive thoughts and behaviours and substance use. Each item asks the child, aged 11-17 years, to rate how much (or how often) he or she has been bothered by the specific symptom during the past 2 weeks. The measure was found to be clinically useful and had good test-retest reliability in the DSM-5 Field Trials conducted in paediatric clinical samples in the USA. ${ }^{33}$ This test is used to screen children aged 12-17 years for mental health issues and substance use. If a participant identifies as having had any substance use in the past 2 weeks, a timeline follow back (TFLB) interview ${ }^{34}$ is administered. The TLFB is a retrospective calendar method of quantifying substance use. It involves asking clients to retrospectively estimate their drug use 7 days to 2 years prior to the interview date.

\section{Resiliency: Child Youth and Resilience Measure (CYRM)}

The CYRM ${ }^{35}$ is a 12-item questionnaire which measures the resources (individual and cultural) available to individuals that may bolster their resilience. The child version is validated for use with children aged 5-9 years and the youth version is validated for use with children aged 9-18.

Perfectionism: Adaptive/Maladaptive Perfectionism Scale (AMPS) The AMPS ${ }^{36}$ has 27 items and measures behaviours associated with perfectionism, such as sensitivity to mistakes, contingent self-esteem, compulsiveness and need for admiration. It is validated for use in children aged 9-12 years, and has been used in adolescents up to age $17 .^{36}$

\section{Symptom validity: Rey FIT}

The Rey 15 item memory test ${ }^{37}$ (FIT) is one of the most commonly used measures to detect malingering or exaggerated symptom reporting. ${ }^{38}$ The test includes presentation of a card with 15 items arranged in three columns by five rows and assesses recall and recognition; it takes approximately $5 \mathrm{~min}$ to administer. Participants thought to be malingering are likely to misjudge the task as more difficult than it actually is and perform more poorly than those with intellectual impairment. ${ }^{39}$ Normative data exists for children 7 years of age and older. ${ }^{40}$

\section{Health economic data}

\section{Medicare benefits scheme (MBS) and pharmaceutical benefits} scheme (PBS) data

To assist with calculating the cost of concussion to our participants, we ask participants for access to their MBS and PBS data. This is an optional component for participation. The data we collect will include information about the date of service, costs to government and patients, the billed item numbers and a description of the type of medical and pharmaceutical services used. As a part of the study, we also collect hospital data (eg, ED costs) and a survey of self-reported patient and family costs (eg, time taken off from work, travel, additional care purchased, missed schooling).

\section{Quality of life for health economics: Child Health Utility 9D (CHU-}

9D)

The CHU-9D is a nine-item measure of health-related quality of life. ${ }^{41}$ The dimensions of the measure assess how worried, sad, tired and annoyed the respondent is, as well as their level of pain, issues with school work/homework, daily routine and ability to join in activities. The CHU-9D parent proxy (currently being validated) allows us to assess children aged 5-7 years. The CHU-9D examines quality of life from a heath economics perspective and will permit future use in cost-utility analyses. 
Data analysis

\section{Population to be analysed}

For prediction of PCS at 2 weeks, 1 month and 3 months postinjury, patients will be included in the analysis if they have complete follow-up data for the specific time point. Children symptomatic at 2 weeks will be defined as 'delayed recoverers'. Our pilot data show $50 \%$ of children are symptomatic at 2 weeks and $26 \%$ at 1 month. ${ }^{19}$

\section{Statistical analysis plan}

Aim 1: identify injury, child and family markers of delayed recovery. We will explore potential mechanisms and clinical predictors of delayed recovery and delayed return to normal activity. The concussion injury (CI) group will be divided into two groups based on PCS status at 2 weeks postinjury: normal recovery and delayed recovery: two or more symptoms over baseline on the PCSI. ${ }^{19}$ These groups will be compared for acute markers: blood biomarkers, MRI, injury factors and preinjury and parent status. The predictive value of each acute marker will be modelled at a bivariate level using logistic regression. Predictors will then be tested together to determine a single, parsimonious multiple logistic regression model. Test fit statistics (Akaike information criterion/Bayesian information criterion/likelihood ratio) will be used to determine model parsimony.

Aim 2: plot and contrast recovery pathways for CI and OI: using CI and OI groups, we will examine recovery trajectories to 3 months postinjury. Our primary outcome is PCS at 2 weeks and 1 month postinjury, adjusting for child age. Secondary outcomes include cognition (Cogsport, Neuropsych screen), physical function and mental health/quality of life and secondary outcome points include T3 (CI only) and T4 (CI and OI).

We will compare group performances from acute presentation in the ED 2 weeks, using longitudinal Generalised Estimating Equation (GEE) models, with post-hoc, multiple comparison adjusted between-group tests at each time point. These modelling techniques allow for more complex data structures and working correlation matrices and account for MAR/MCAR missing data.

Aim 3: estimate costs of child concussion and factors predicting high cost. A cost-of-illness study will estimate costs of child concussion. Costs include: assessment/ treatment (ED, outpatient, general practice, pharmaceutical), carers (time off work/usual duties, additional care purchased, travel) and lost schooling (missed school days, cost of education per day). Data from aim 1 will be drawn on to match costs with children's recovery pathways. Regression modelling will be used to predict high-cost patients using data from assessment, patient demographics, time to presentation, nature of injury, involvement in sport/sport type, comorbidities/other injuries, prior concussions and medical history.

\section{Sample size estimation}

To determine statistically and clinically meaningful differences between CI and OI groups (aims 2, 3) and maintain power for within-CI analyses (aim 1), more participants are required in the CI group than the OI group. For betweengroup analyses, incorporating $10 \%$ attrition (based on pilot data) per wave, to find a difference of 0.3 of a SD, with $80 \%$ power and an error level of 0.05 , a sample of CI $n=400$ and OI $n=125$ is required (with attrition, CI $n=236$, OI $n=73$ at T4). GEE/regressions models, including an interaction with potential confounder child age (dichotomous: 5-11, 12-18), will detect a small effect size of $\eta^{2}=0.03$. With a CI sample of $n=400$, exploratory/repeated GEE/regression models for aims 1 and 2 will have adequate power to detect small-to-medium effect sizes.

\section{Methodological considerations \\ Identifying clinically significant concussions}

The pilot study for the Take CARe protocol highlighted important methodological considerations, one of them being the difficulties associated with determining a clinically significant concussion using a standardised instrument (ie, the PCSI). The PCSI is a valid and reliable measure for identifying the range and severity of concussion symptoms. ${ }^{14}$ The definition we formerly used to determine clinical significance of concussion was the presence of three or more symptoms on the PCSI rated as worse than the baseline scores. ${ }^{42}$ We have adopted a modified and validated ${ }^{19}$ definition with increased sensitivity, using two or more symptoms on the PCSI rated as worse compared with baseline, consistent with analysis undertaken from our pilot protocol. ${ }^{19}$

\section{Bias in recruitment}

The nature of the pilot study required a significant time commitment from all enrolled participants. Parents and children were asked to attend the hospital on at least three separate occasions, possibly four, for approximately 1 hour each visit. Many eligible participants who were approached in the ED expressed interest in participating, but declined participation due to competing time commitments. It is therefore possible we were recruiting a biased sample (eg, families with only one parent in fulltime employment, greater family support, higher socioeconomic status, geographical proximity to RCH). To address this issue in the new protocol, we have created an online component using the REDCap ${ }^{43}$ data collection service, where participants may complete an abbreviated battery of questionnaires at home for all four follow-up time points, with their responses monitored by research staff for clinically significant results. In addition to this, there is also an option for a further abbreviated phone option, where study participants are called and asked a brief battery of questions that are of most relevance to the study aims at the 1-4 days and 2 week time points.

\section{Single site}

The present study is being conducted at a single site, the RCH (a paediatric tertiary hospital). While the presentation of concussion is significant at RCH ( 1000 per year), it is possible that this will limit the recruitment options relative to other studies (eg, A-CAP) ${ }^{44}$ and reduce statistical power. 


\section{ETHICS AND DISSEMINATION}

Ethical approval was obtained through the RCH Human Research Ethics Committee (33122). Written parental consent is obtained for all participants and written participant consent is obtained for participants aged 13 years and older. All participants can withdraw consent at any time and younger participants are asked to assent to all aspects of the study (eg, MRI). The study poses little to no risk to participants and their families. Written informed consent is obtained from all participating families. Participation in the study does not interfere with the typical care patients receive in the ED (eg, CT head). Clinical follow-up is provided by clinicians in the concussion clinic at all time points and, if necessary, participants are referred to other clinics for additional management (eg, rehabilitation, neurosurgery, clinical psychology). All children under 12 years old enrolled in the MRI stream of the study are offered a mock scan before their full MRI takes place. Children 13 years and over have the right to refuse MRI and/or bloods even if their parents' consent on their behalf.

Results from this study will be disseminated at regional and international conferences, in peer-reviewed journals and via popular media (eg, social media).

\section{SIGNIFICANCE AND OUTLOOK}

Child concussion is a major health problem of significant cost and concern to parents, schools, sporting association and the wider community. Recent media and academic ${ }^{44}$ focus has heightened awareness of potential risks for concussion, to the extent of consideration of excluding children from contact sports. A high proportion of children are at risk of poor recovery and we cannot yet explain 'why' or identify 'who'. This lack of understanding of recovery from concussion and premature return to sports and school puts children at significant risk of further injury, ${ }^{6}$ including repeated concussion. Better understanding of recovery pathways and timelines, and more accurate, developmentally informed measurement of symptoms is critical. Clinicians, coaches, teachers and parents need this information on which to base decisions about return to school and sports following concussion.

Our findings will help to:

- Establish the incidence and characteristics of delayed recovery following child concussion.

- Employ a multimodal approach to identify acute markers of PCS, investigate synergies, define clinical phenotype of injuries and incorporate them into diagnostic and assessment protocols, facilitating early and tailored intervention.

- Provide an understanding of the economic impact of child concussion for policymakers.

\footnotetext{
Author affiliations

${ }^{1}$ Clinical Sciences, Murdoch Children's Research Institute, Melbourne, Victoria, Australia

${ }^{2}$ School of Psychological Sciences, University of Melbourne, Melbourne, Victoria, Australia

${ }^{3}$ Department of Paediatrics, University of Melbourne, Carlton, Victoria, Australia
}

${ }^{4}$ Emergency Department, Royal Children's Hospital, Melbourne, Victoria, Australia ${ }^{5}$ Department of Women's and Children's Health, University of Padova, Padova, Italy ${ }^{6}$ Centre for Health Policy, Melbourne School of Population and Global Health,

University of Melbourne, Melbourne, Victoria, Australia

${ }^{7}$ Department of Neurosurgery, Austin and Cabrini Hospitals, Parkville, Victoria, Australia

${ }^{8}$ Department of Rehabilitation Medicine, Royal Children's Hospital, Melbourne, Victoria, Australia

${ }^{9}$ Psychology Service, Royal Childrens Hospital, Melbourne, Victoria, Australia

Contributors FEB, SB, KD, GAD, SJCH, VI, MS, MT and VA conceptualised and designed the study and drafted the initial manuscript. NA, CJC, AC, MD, KD, CG, GP, VR, EJT and KT made substantial contributions to the conception of the study and critically revising the manuscript for important intellectual content. All authors approved the final manuscript as submitted and agree to be accountable for all aspects of the work

Funding This work was supported by the Royal Children's Hospital Research Foundation.

Competing interests GAD is an honorary member of the Australian Football League Concussion Working Group and has attended meetings organized by sporting organisations including the National Football League (NFL; USA), National Rugby League (Australia), and FIFA (Switzerland); however, he has not received any payment, research funding or other monies from these groups other than for travel costs. All authors declare that they have no financial relationships relevant to this article to disclose.

Patient consent for publication Not required.

Ethics approval Royal Children's Hospital Human Research Ethics Committee.

Provenance and peer review Not commissioned; externally peer reviewed.

Open access This is an open access article distributed in accordance with the Creative Commons Attribution Non Commercial (CC BY-NC 4.0) license, which permits others to distribute, remix, adapt, build upon this work non-commercially, and license their derivative works on different terms, provided the original work is properly cited, appropriate credit is given, any changes made indicated, and the use is non-commercial. See: http://creativecommons.org/licenses/by-nc/4.0/.

\section{REFERENCES}

1. McCrory P, Meeuwisse W, Dvořák J, et al. Consensus statement on concussion in sport - the 5th international conference on concussion in sport held In Berlin, October 2016. Br J Sports Med 2017:11.

2. Crowe L, Babl F, Anderson V, et al. The epidemiology of paediatric head injuries: data from a referral centre in Victoria, Australia. $J$ Paediatr Child Health 2009;45:346-50.

3. Babl FE, Borland ML, Phillips N, et al. Accuracy of PECARN, CATCH, and CHALICE head injury decision rules in children: a prospective cohort study. Lancet 2017;389:2393-402.

4. Meehan WP, Mannix R. Pediatric concussions in United States emergency departments in the years 2002 to 2006. J Pediatr 2010;157:889-93.

5. Graves JM, Rivara FP, Vavilala MS. Health care costs 1 year after pediatric traumatic brain injury. Am J Public Health 2015;105:e35-41.

6. Davis GA, Anderson V, Babl FE, et al. What is the difference in concussion management in children as compared with adults? A systematic review. Br J Sports Med 2017;51:949-57.

7. Zemek R, Barrowman N, Freedman SB, et al. Clinical risk score for persistent postconcussion symptoms among children with acute concussion in the ED. JAMA 2016;315:1014-25.

8. Truss K, Godfrey C, Takagi M, et al. Trajectories and risk factors for post-traumatic stress symptoms following pediatric concussion. $J$ Neurotrauma 2017;34:2272-9.

9. Giza CC, Hovda DA. The neurometabolic cascade of concussion. $J$ Athl Train 2001;36:228.

10. Guskiewicz KM. Assessment of postural stability following sportrelated concussion. Curr Sports Med Rep 2003;2:24-30.

11. Lugones M, Parkin G, Bjelosevic S, et al. Blood biomarkers in paediatric mild traumatic brain injury: a systematic review. Neurosci Biobehav Rev 2018;87:206-17.

12. Bressan S, Takagi M, Anderson V, et al. Protocol for a prospective, longitudinal, cohort study of postconcussive symptoms in children: the Take C.A.Re (Concussion Assessment and Recovery Research) study. BMJ Open 2016;6:e009427.

13. McCrory $P$, Meeuwisse $W H$, Aubry $M$, et al. Consensus statement on concussion in sport: the 4th International Conference on 
Concussion in Sport held in Zurich, November 2012. Br J Sports Med 2013:47:250-8.

14. Gioia GA, Schneider JC, Vaughan CG, et al. Which symptom assessments and approaches are uniquely appropriate for paediatric concussion? Br J Sports Med 2009;43(Suppl 1):113-22.

15. Babl FE, Dionisio D, Davenport L, et al. Accuracy of components of SCAT to identify children with concussion. Pediatrics 2017;140:e20163258.

16. Echemendia RJ, Meeuwisse W, McCrory P, et al. The Sport Concussion Assessment Tool 5th Edition (SCAT5): Background and rationale. Br J Sports Med 2017;51:848-50.

17. Davis GA, Purcell L, Schneider KJ, et al. The Child Sport Concussion Assessment Tool 5th Edition (Child SCAT5). Br J Sports Med 2017;39:bjsports-2017-097492.

18. Hicks CL, von Baeyer CL, Spafford PA, et al. The faces pain scalerevised: toward a common metric in pediatric pain measurement. Pain 2001;93:173-83.

19. Hearps SJ, Takagi M, Babl FE, et al. Validation of a score to determine time to postconcussive recovery. Pediatrics 2017;139:e20162003.

20. Collie A, Maruff P, Makdissi M, et al. CogSport: reliability and correlation with conventional cognitive tests used in postconcussion medical evaluations. Clin J Sport Med 2003;13:28-32.

21. Wechsler D. Wechsler Abbreviated Scale of Intelligence. 2nd edn. San Antonio: TX Pearson, 2011

22. Wechsler D. Wechsler preschool and primary scale of intelligence. 4th edn. San Antonio, TX: The Psychological Corporation, 2012.

23. Wechsler D. Wechsler intelligence scale for children, fourth edition (WISC-IV). San Antonio: TX: The Psychological Corporation, 2003.

24. Anderson $\mathrm{P}$, Anderson $\mathrm{V}$, Northam $\mathrm{E}$, et al. Standardization of the Contingency Naming Test (CNT) for school-aged children: A measure of reactive flexibility. Clinical Neuropsychological Assessment 2000;1:247-73.

25. Broglio SP, Puetz TW. The effect of sport concussion on neurocognitive function, self-report symptoms and postural control : a meta-analysis. Sports Med 2008;38:53-67.

26. Rey A. L'examen psychologique dans les cas d'encéphalopathie traumatique. Archives de Psychologie 1941;28:21.

27. Lovell MR, Solomon GS. Neurocognitive test performance and symptom reporting in cheerleaders with concussions. J Pediatr 2013;163:1192-5.

28. Benton A, Sd H, Sivan A. Multilingual aplasia examination. 2nd edn. lowa City, IA: AJA Associates, 1983.

29. Achenbach T. Child behavior checklist:ASEBA. 2001.

30. Foa EB, Johnson KM, Feeny NC, et al. The child PTSD symptom scale: a preliminary examination of its psychometric properties. Journal of Clinical Child \& Adolescent Psychology 2001;30:376-84.

31. Varni JW, Burwinkle TM, Katz ER, et al. The PedsQL in pediatric cancer: reliability and validity of the pediatric quality of life inventory generic core scales, multidimensional fatigue scale, and cancer module. Cancer 2002;94:2090-106.

32. Kessler R. Kessler Psychological Distress Scale. Psychological Medicine 2002;32:949-56.

33. Narrow WE, Clarke DE, Kuramoto SJ, et al. DSM- 5 field trials in the United States and Canada, Part III: development and reliability testing of a cross-cutting symptom assessment for DSM-5. Am J Psychiatry 2013;170:71-82.

34. Sobell L, Sobell M, Followback T. A calendar method for assessing alcohol and drug use. Toronto, Ontario: Addiction Research Foundation, 1996.

35. Liebenberg L, Ungar M, LeBlanc JC. The CYRM-12: a brief measure of resilience. Can J Public Health 2013;104:e131-5.

36. Rice K, Preusser K. The Adaptive/Maladaptive Perfectionism Scale. Measurement and evaluation in counseling and development. 2002;34:210-22.

37. Rey A. L'examen clinique en psychologie. Paris, France: Presses Universitaires de France, 1964.

38. Slick DJ, Tan JE, Strauss EH, et al. Detecting malingering: a survey of experts' practices. Arch Clin Neuropsychol 2004;19:465-73.

39. Lezak MD, Howieson DB, Loring DW. Neuropsychological assessment. 4th edn. New York: Oxford University Press, 2004.

40. Strauss E, Sherman EMS, Spreen O. A compendium of neuropsychological tests: administration, norms, and commentary: Oxford University Press, 2006.

41. Stevens K. Assessing the performance of a new generic measure of health-related quality of life for children and refining it for use in health state valuation. Appl Health Econ Health Policy 2011;9:157-69.

42. Zemek R, Osmond MH, Barrowman N. Pediatric Emergency Research Canada (PERC) Concussion Team. Predicting and preventing postconcussive problems in paediatrics (5P) study: protocol for a prospective multicentre clinical prediction rule derivation study in children with concussion. BMJ Open 2013;3:e003550.

43. Harris PA, Taylor R, Thielke R, et al. Research electronic data capture (REDCap) - a metadata-driven methodology and workflow process for providing translational research informatics support. J Biomed Inform 2009;42:377-81.

44. Yeates KO, Beauchamp M, Craig W, et al. Advancing Concussion Assessment in Pediatrics (A-CAP): a prospective, concurrent cohort, longitudinal study of mild traumatic brain injury in children: protocol study. BMJ Open 2017;7:e017012. 